\title{
Tissue-Specific Effects of Bariatric Surgery Including Mitochondrial Function
}

\author{
Simon N. Dankel, ${ }^{1,2}$ Vidar Staalesen, ${ }^{1}$ Bodil Bjørndal, ${ }^{1}$ Rolf K. Berge, ${ }^{1}$ Gunnar Mellgren, ${ }^{1,2}$ \\ and Lena Burri ${ }^{1}$
}

${ }^{1}$ Institute of Medicine, Haukeland University Hospital, University of Bergen, 5021 Bergen, Norway

${ }^{2}$ Hormone Laboratory, Haukeland University Hospital, 5021 Bergen, Norway

Correspondence should be addressed to Gunnar Mellgren, gunnar.mellgren@med.uib.no

Received 2 September 2010; Accepted 14 December 2010

Academic Editor: Francesco Saverio Papadia

Copyright (C) 2011 Simon N. Dankel et al. This is an open access article distributed under the Creative Commons Attribution License, which permits unrestricted use, distribution, and reproduction in any medium, provided the original work is properly cited.

\begin{abstract}
A better understanding of the molecular links between obesity and disease is potentially of great benefit for society. In this paper we discuss proposed mechanisms whereby bariatric surgery improves metabolic health, including acute effects on glucose metabolism and long-term effects on metabolic tissues (adipose tissue, skeletal muscle, and liver) and mitochondrial function. More short-term randomized controlled trials should be performed that include simultaneous measurement of metabolic parameters in different tissues, such as tissue gene expression, protein profile, and lipid content. By directly comparing different surgical procedures using a wider array of metabolic parameters, one may further unravel the mechanisms of aberrant metabolic regulation in obesity and related disorders.
\end{abstract}

\section{Introduction}

Bariatric surgery represents a highly successful treatment strategy for obesity and secondary diseases such as type 2 diabetes mellitus (T2DM), at least in morbidly obese patients [1]. Though similar effects may be obtained with lifestyle intervention [2], many morbidly obese patients do not succeed in making sufficient permanent lifestyle changes [3]. The success rate of surgery varies depending on the surgical procedure and individual factors including lifestyle/nutrition, age, gender, and genetics/epigenetics $[4,5]$. Besides being an effective treatment for obesity that decreases mortality and morbidity, bariatric surgery confers some health risk including renal stone formation and oxalate nephropathy (calcium oxalate crystals in the kidney) [6]. Due to changes to the gastrointestinal tract with malabsorptive surgery, absorption of vitamins and minerals is affected and bariatric surgery patients are advised to take micronutrient supplements [7].

It is of utmost importance to fully understand the metabolic changes induced by bariatric surgery, as it may lead to novel treatment strategies for obesity and related health problems. Because morbidly obese patients undergoing bariatric surgery effectively and consistently lose excess body weight and reduce obesity-related comorbidity, they represent a very useful patient group for studying mechanisms that regulate metabolic health. The most common surgical procedures are today performed laparoscopically and include adjustable gastric band (LAGB), sleeve gastrectomy (LSG), Roux-en-Y gastric bypass (RYGB), and biliopancreatic diversion (BPD). BPD often includes duodenal switch (BPD/DS) and sleeve gastrectomy. RYGB and BPD show the best long-term results in terms of fat loss $[8,9]$ and diabetes resolution [1]. Whereas LAGB and LSG exert their effects through reduced ventricular volume and food intake, RYGB and BPD (with sleeve gastrectomy) combine this effect with malabsorption of nutrients by means of bypassing a substantial part of the small intestine. In addition, the intestinal reconfiguration results in a rapid improvement of diabetes within days in most patients, which cannot be entirely ascribed to energy restriction or fat loss [10]. This intriguing observation has led to the hypothesis that 
regulatory factors in the small intestine, including peptide hormones and nerve signals, are critical in modulating glucose homeostasis. Thus, the metabolic effects of bariatric surgery are both dependent and independent of fat loss.

In the present paper, our overall aim was to describe central mechanisms that may mediate the beneficial effects of bariatric surgery on metabolic health. Our specific objective was first to summarize the most important findings regarding fat loss-independent effects of bariatric surgery. Because of the scarcity of data on the acute effects of surgery on various metabolic parameters, we also discuss longerterm metabolic effects that may result from a combination of fat loss and intestinal surgery, including effects on mitochondria. Finally, our objective was to provide new perspectives for future research regarding the metabolic effects of bariatric surgery.

\section{Acute Metabolic Effects of Bariatric Surgery}

In the weeks following bariatric surgery, patients are limited to consumption of liquids and their nutrient intake becomes drastically reduced. Thus, when evaluating the metabolic effects of bariatric surgery, one must consider the isolated impact of reduced energy intake versus weight loss. In healthy lean subjects, energy restriction may induce a "starvation diabetes" marked by hepatic and peripheral insulin resistance [11]. In obese individuals, on the other hand, energy restriction may improve glycemic control and insulin sensitivity independently of weight loss during the first days [12]. The metabolic effects of weight loss may begin to be substantial after 7-10 days of energy restriction [13]. In diabetic obese individuals, four days of energy restriction improved hepatic insulin sensitivity leading to suppressed hepatic glucose output and reduced fasting glucose levels [14].

However, energy restriction cannot fully account for the acute improvement in glycemic control after intestinal bypass. Studies comparing intestinal bypass (RYGB) to energy-restrictive procedures, both of which require patients to consume only liquids in the first weeks postsurgery, show a specific acute effect on glucose metabolism after RYGB. In contrast to RYGB $[5,15]$, the LAGB procedure showed no amelioration of diabetes until a considerable degree of weight loss had occurred in a randomized controlled trial of obese diabetic patients [16]. Kashyap et al. showed that only RYGB and not LSG improved postprandial insulin secretion and sensitivity despite similar reductions in weight and fasting insulin levels (subjects were compared 1-3 weeks before versus 1 week after surgery) [17]. Similarly, a randomized controlled trial of diabetic patients by Peterli et al. showed that RYGB but not LSG increased meal-induced insulin responses 1 week postsurgery, while effects of the different surgical procedures were similar at 3 months, when similar weight loss was achieved [18]. These observations clearly point towards mechanisms of acute glucose control that are unique to intestinal bypass and that are independent of energy restriction and weight loss.

The acute effects of bariatric surgery on glycemic control and metabolic health likely involve a combination of several factors, including insulin secretion, peripheral insulin sensitivity, and hepatic insulin sensitivity. Distinct effects have been observed with the BPD and RYGB procedures. The effects of BPD are more due to intestinal reconfiguration and malabsorption, while RYGB involves a greater restriction of gastric volume (to $30 \mathrm{ml}$ versus $300-400 \mathrm{ml}$ with BPD). BPD rapidly improved insulin sensitivity along with a reduction in insulin secretion and normalization of blood glucose levels 1 week [19] as well as 1 month postsurgery, measured by euglycemic-hyperinsulinemic clamp [20]. On the other hand, RYGB did not improve peripheral insulin sensitivity 2 weeks or 1 month postsurgery, also measured by euglycemic-hyperinsulinemic clamp [21, 22]. Rather, RYGB may acutely reduce hyperglycemia by increasing foodinduced insulin secretion via increased release of incretins $[18,23]$, in some cases resulting in episodes of hypoglycaemia $[24,25]$. It should be noted that another study of RYGB patients before versus 6 days after surgery found significant improvement in peripheral insulin sensitivity measured by intravenous glucose tolerance tests and homeostatic model assessment (HOMA) [15]. However, euglycemichyperinsulinemic clamp is a superior technique for measuring insulin resistance. It is possible that improvements in hepatic rather than peripheral insulin sensitivity may represent a key mechanism of glucose control after RYGB. A study in mice suggested that restoration of glucose control after RYGB may be due to reduced endogenous glucose output via improved hepatic insulin sensitivity, and also altered intestinal gluconeogenesis [26].

The proposed hypotheses attempting to explain the surgery-specific effects on glucose metabolism remain speculative. Various mechanisms are likely to contribute [10]. The lower intestinal (hindgut) hypothesis proposes that exclusion of the proximal intestine leads to a more rapid delivery of nutrients to the lower intestine, resulting in increased release of gut hormones that regulate glucose metabolism (e.g., glucagon-like peptide-1 (GLP-1), peptide Y (PYY), and oxyntomodulin secreted by L-cells in the distal small intestine and parts of the colon) $[27,28]$. It is possible that central effects of GLP-1 may contribute to reducing hepatic insulin resistance and glucose production [29]. Importantly, a stimulatory effect on the circulating levels of incretins such as GLP-1 and glucose-dependent insulinotropic polypeptide (GIP), which augment insulin secretion, was not observed with energy restriction in diabetic patients [23]. It is interesting to note that secretion of GIP, which is produced in K-cells in the proximal intestine, was found to be increased after RYBG [27] but decreased after BPD [19, 20]. GIP has been shown to promote energy storage in adipose tissue, and inactivation of GIP in mice was shown to improve insulin resistance [30]. Moreover, the upper intestinal (foregut) hypothesis postulates that the proximal intestine that is bypassed with RYGB and BPD releases one or more factors with adverse effects on glucose metabolism. In polygenic diabetic rats (Goto-Kakizaki), Rubino et al. found that duodenal-jejunal bypass enhanced glucose metabolism independently of food intake and body weight [31]. The mechanism may involve reduced nutrient contact with the excluded duodenum [32]. In diabetic humans with a $60 \mathrm{~cm}$ 
flexible plastic sleeve inserted to inhibit nutrient contact with duodenal mucosa (endoluminal duodenal sleeve), glycemic control both after and between meals improved considerably already after 1 week without significant weight loss [33].

Neuronal signalling has been implicated as a critical factor in the postsurgery control of glucose metabolism. The vagus nerve is a critical regulator of the digestive process as well as of appetite regulation in the gut-brain-gut axis, and vagotomy (resection of the vagus nerve) has been used to treat obesity [34]. More recently, blockage of vagus activity by an implanted medical device has shown promising effects on weight loss in humans [35]. Randomized controlled trials are needed to confirm this effect, and possible acute effects on glucose metabolism would be of particular interest. An acute effect of vagotomy to reduce insulin secretion was demonstrated several decades ago in rats [36]. Hepatic glucose production is centrally regulated via the hepatic branch of the vagus nerve [37]. Thus, vagus nerve function may at least partially mediate the acute effects of intestinal bypass surgery on glucose metabolism.

\section{Long-Term Effects on Insulin Sensitivity, Glucose Metabolism, and Metabolic Tissues}

While it is difficult to isolate the fat loss-dependent and independent effects of bariatric surgery, long-term studies of bariatric patients indicate that a greater loss of excess body weight associates with an improved effect on diabetes [1]. When performed in morbidly obese patients, the RYGB and $\mathrm{BPD} / \mathrm{DS}$ procedures often reduce body weight to about half the original weight within the two first years, followed by a stable weight for at least 10 years $[8,9]$. Although bariatric patients usually remain obese after surgery with a bodymass index (BMI) above 30, their metabolic profile is in many respects similar to that of healthy, lean individuals. A prospective study of whole body and muscle insulin sensitivity before and one year after bariatric surgery found that weight-stable RYGB patients were more comparable to lean than weight-matched controls [38]. However, this beneficial effect of fat loss is not specific to bariatric surgery, since shorter-term nutritional intervention and exercise have also been shown to induce weight loss-independent improvements in glucose homeostasis [39, 40]. Nonetheless, bariatric surgery allows for relatively controlled long-term prospective studies of both moderate and profound fat loss and has provided important insight into the altered functions of metabolic tissues in (morbid) obesity.

\subsection{Bariatric Surgery and the Functions of Adipose Tissue and} Skeletal Muscle. Adipose tissue performs functions that are critical to metabolic health, providing a storage buffer for surplus energy and secreting peptide hormones, cytokines, lipids and other molecules, thereby coordinating metabolic regulation with other organs [41]. Obesity is characterized by increased fat mass and altered adipose tissue function, involving cellular stress responses, changes in extracellular matrix, infiltration of immune cells such as macrophages, chronic inflammation, and potential aberration of adipogenesis, angiogenesis, and tissue remodelling. These changes are related to increased ectopic lipid accumulation and secretion of adipokines with potential adverse systemic effects [42, 43].

Bariatric surgery may improve adipose tissue function via various mechanisms, including an acute reduction in energy intake, changes in the endocrine and immune-related functions of the gut, and profound reductions in adipose tissue mass [44]. There is a paucity of information on the acute effects of bariatric surgery on adipose tissue function in humans. In cultured human subcutaneous adipocytes, GIP together with insulin was found to activate lipoprotein lipase [45], suggesting that altered GIP levels after bariatric surgery may affect lipid uptake in adipose tissue [46].

Several long-term studies of bariatric patients have revealed important insights into the alterations of adipose tissue function in obesity. Endoplasmic reticulum stress is increased in obesity, markers of which were strongly reduced in both subcutaneous adipose tissue and liver tissue 1 year after RYGB [47]. Moreover, a reduction in macrophage infiltration and the expression of chemoattractant and proinflammatory genes was observed in a global gene expression study of subcutaneous adipose tissue before versus 3 months after RYGB [48]. Our recent microarray study of subcutaneous adipose tissue before versus 1 year after BPD/DS corroborates these findings, showing a substantial reduction in genes related to immunity and defense functions [49]. These studies have also demonstrated that bariatric surgery, most likely due to the profound fat loss, strongly alters the expression of genes involved in extracellular matrix functions $[49,50]$. While similar observations have been made in dietary intervention studies $[51,52]$, the differential gene expression after bariatric surgery may be specific to the extreme degree of fat loss and possibly also some effects of the gastrointestinal changes after surgery.

Muscle insulin resistance may be a critical factor in the pathogenesis of diabetes and metabolic complications including fatty liver [53]. Bariatric surgery was found to decrease intramyocellular lipids measured by Oil-red-O staining 3 and 9 months after RYGB or LAGB [54]. The study also found reduced gene expression of SCD1 and $P D K 4$ in skeletal muscle after 3 months, whereas $P P A R \alpha$, $M C A D, C P T 1$, and UCP3 were down-regulated only at the 9-month time point. Greco et al. showed a significant reduction in intramyocellular lipids 6 months after BDP [55]. A magnetic resonance spectroscopy study also found significant reductions in intramyocellular lipids already 1 month after BPD [56], while this early effect was not reported in a study of seven RYGB patients [57]. Further studies should be performed to verify whether there is a surgeryspecific effect on intramyocellular lipids. It is tempting to speculate whether a reduced circulating level of GIP specifically after BPD may play a role, since suppression of GIP may reduce lipid storage in skeletal muscle and liver as in adipose tissue [46].

3.2. Bariatric Surgery and Liver Function. The liver is a critical organ for maintaining metabolic homeostasis in the body. Lipid homeostasis is typically disrupted in obese 
individuals, reflected by chronically elevated plasma lipid levels. Together with adipose tissue, the liver is the main lipidemic organ during systemic hyperlipidimia, and the development of insulin resistance and diabetes [58]. The liver takes up free fatty acids (FFAs) released from adipose tissue, as well as circulating triglycerides (TGs). The FFAs are either degraded by $\beta$-oxidation or repackaged to TGs in very low-density lipoprotein particles and released into the bloodstream.

Steatosis (fatty liver) occurs when there is an imbalance between lipogenesis and fatty acid oxidation. When the rate of hepatic fatty acid uptake from plasma and de novo fatty acid synthesis predominates over the rate of fatty acid oxidation and triglyceride export, triglycerides will be deposited in the liver. The resulting liver injury is often associated with obesity, and $95 \%$ of individuals with class III obesity (BMI $\geq 40 \mathrm{~kg} / \mathrm{m}^{2}$ ) have alterations in routine liver biopsies due to steatosis, steatohepatitis, or fibrosis [59]. These liver abnormalities are known as nonalcoholic fatty liver disease (NAFLD) which increases the risk of developing T2DM, dyslipidemia, and hypertension [60]. It is unknown whether alterations in hepatic glucose, FA, and lipoprotein metabolism and inflammation are causes of NAFLD, or whether these abnormalities are increased in the presence of NAFLD. Increased hepatic $\beta$-oxidation will generate reactive oxygen species and promote the development from steatosis to the inflammatory stage nonalcoholic steatohepatitis (NASH). Reduced lipid storage efficiency in adipose tissue and increased rates of adipose tissue lipolysis in obesity promote ectopic lipid accumulation, challenging the liver with high amounts of FFAs [43].

The amelioration of NAFLD and liver function by bariatric surgery may be an important contributor to the systemic improvement of energy homeostasis. Most studies have shown improved liver histology and liver function as well as insulin sensitivity in obese subjects with NAFLD and NASH after bariatric surgery [61]. The weight loss and reduced energy overload improve liver parameters, and the less severe steatosis is fully reversible. In accordance with this, LAGB changed the hepatic adipokine levels of NAFLD patients in an anti-inflammatory direction, increased the adiponectin protein level, and decreased the leptin receptor mRNA level 6 months after surgery [62]. Another study found that a gene with a central role in lipid peroxidation, CYP2E1, was significantly reduced in liver after weight loss following bariatric surgery [63]. Hepatic lipid peroxidation, as measured by the malondialdehyde (MDA) level, was reduced and liver steatosis decreased from $17 \%$ prior to surgery to $2 \%$ several months after. These studies show that bariatric surgery can improve the more progressed NASH disease. The amelioration of NAFLD and liver function by bariatric surgery may be an important contributor to the systemic improvement of energy homeostasis. However, the lack of randomized clinical trials so far makes it difficult to conclude on the use of bariatric surgery for treatment of NASH [64].

Intriguingly, no reduction in intrahepatocellular lipid levels was observed 1 month after RYGB while insulin sensitivity markedly improved [57]. This was surprising given the strong link between hepatic lipid levels and insulin resistance. On the other hand, hepatic lipid content was markedly reduced by 6 and 12 months. Of note, neither visceral adipose tissue mass nor intramyocellular lipid levels correlated with the improvements in insulin sensitivity. These observations should be validated in short-term randomized studies with increased power and in subjects undergoing different forms of bariatric surgery. This may provide important new insight into the mechanisms whereby bariatric surgery affects the systemic metabolic homeostasis via the individual metabolic tissues. Development of insulin resistance in the liver may also partly result from the altered secretion of metabolic and inflammatory adipokines from adipose tissue $[58,65]$. The general metabolic effects of bariatric surgery are summarized in Figure 1.

\section{Mitochondria in Obesity and Diabetes Mellitus}

4.1. Mitochondria before Bariatric Surgery. Mitochondrial metabolism is essential in maintaining normal physiological function in human cells, for example, by providing energy in the form of ATP and performing fatty acid (FA) oxidation. These metabolic functions are reduced in insulin-responsive tissues (muscle and adipose tissue) in obesity and T2DM. No consensus has been reached so far whether insulin resistance is a result of reduced mitochondrial density and whether it is the cause or consequence of mitochondrial dysfunction [66-76]. In general, insulin regulates protein synthesis, glycolysis, and glucose storage in muscle and liver, lipid synthesis, and storage in liver and adipose tissue, and inhibits gluconeogenesis and ketogenesis in liver [77]. In particular, insulin signalling was shown to influence mitochondrial DNA and protein synthesis and affect mitochondrial respiration and ATP production [78]. Factors that may cause mitochondrial dysfunction include genetic defects, age, physical inactivity, and nutritional overload. These factors may exert changes in mitochondrial size and content, activity and coupling of mitochondrial respiration, copy numbers of the mitochondrial genome, reactive oxygen species (ROS) production, FA oxidation, and more.

Altered gene expression and protein levels may reflect the status of mitochondrial function in obesity and related diseases. Reduced expression of specific genes in myocytes was proposed to result in mitochondrial dysfunction in patients with T2DM. For example, the expression of several genes encoding enzymes involved in the electron transport chain was reduced in muscle of family history-positive nondiabetic subjects and subjects with T2DM [79, 80]. However, this reduced mRNA expression was not reflected in a reduced respiratory rate per mitochondrion in insulinresistant muscle [66]. The expression of several genes of the FA oxidation pathway was also decreased in skeletal muscle in T2DM [80]. Moreover, genes related to mitochondrial biogenesis, such as peroxisome proliferator-activated receptor $\gamma$ coactivator-1 (PGC-1 $\alpha$ and $P G C-1 \beta)$, were similarly downregulated in diabetes $[79,80]$. Another study attributed the decreased skeletal muscle FA oxidation in obesity to 


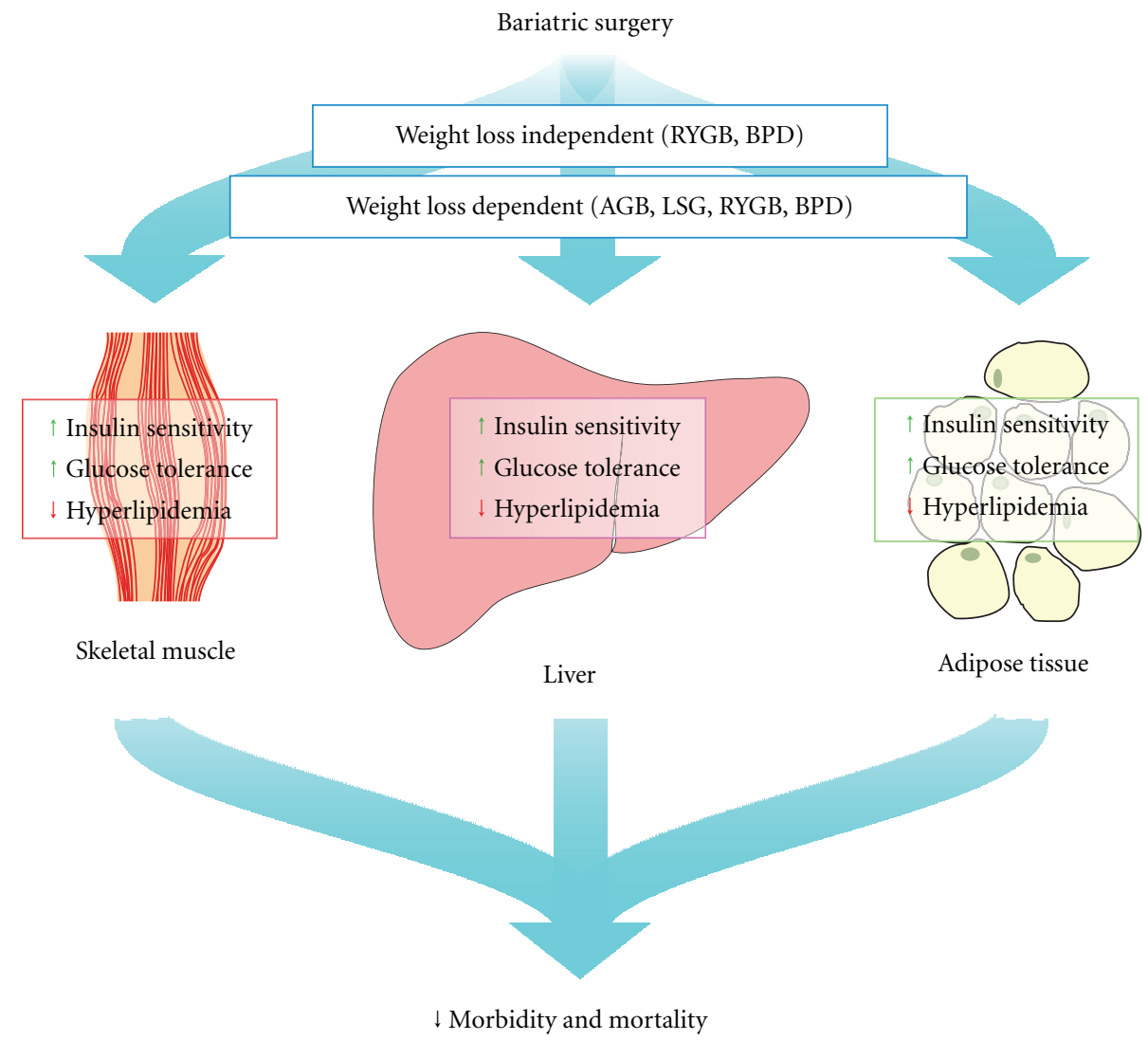

FIGURE 1: Scheme of the metabolic changes in response to adjustable gastric band (AGB) and laparoscopic sleeve gastrectomy (LSG), whose metabolic effects are mainly dependent on weight loss, and Roux-en-Y gastric bypass (RYGB) and biliopancreatic diversion (BPD), whose metabolic effects are also independent of weight loss.

reduced mitochondrial content (and not to intrinsic mitochondrial defects), but showed no reduction in the protein levels of PGC- $1 \alpha, \mathrm{PGC}-1 \beta$, peroxisome proliferator-activated receptor $\alpha(\operatorname{PPAR} \alpha)$, or the mitochondrial transcription factor A (TFAM). Instead, the protein level of PPAR $\gamma$ was increased, possibly due to decreased FA oxidation [81]. Furthermore, it has been proposed that mitochondrial fusion and metabolism in obese and non-obese T2DM patients is impaired due to reduced expression of mitofusin 2 (MFN2) in skeletal muscle [82-84]. MFN2 is an outer mitochondrialand endoplasmic reticulum membrane protein with fusion activity important for mitochondrial dynamics and morphology [83-85]. MFN2 transcriptional activation is regulated by both PGC- $1 \alpha$ and $\beta$ and the estrogen-related receptor $\alpha(\operatorname{ERR} \alpha)$. More studies are needed to delineate the molecular mechanisms responsible for altered mitochondrial function in obesity.

It is of potential interest to compare different adipose tissue depots regarding mitochondrial function. Increased visceral (intra-abdominal) adipose tissue mass is particularly associated with risk of metabolic disease relative to subcutaneous fat. Diabetic humans show decreased expression of respiratory rate genes in visceral adipose tissue compared to healthy humans [87]. It has also been shown that visceral adipose tissue contains twice as many, but smaller, mitochondria per milligram of tissue than the subcutaneous depot [87]. This resulted in visceral fat eliciting lower mitochondrial respiration than subcutaneous fat, when expressed per cell. However, per milligram tissue visceral fat was metabolically more active than subcutaneous fat.

4.2. Mitochondrial Regulatory Pathways and Biogenesis after Bariatric Surgery. Mitochondrial function is destabilized in obesity and T2DM, but few studies have specifically investigated the effect of bariatric surgery on mitochondrial metabolism in myocytes and adipocytes. Bariatric surgery has been shown to induce severalfold changes in the expression of genes encoding proteins involved in mitochondrial function and biogenesis in muscle and adipose tissue. In a global gene expression study of subcutaneous adipose tissue before versus 1 year after BPD/DS [49], we observed an altered expression of certain genes involved in the mitochondrial electron transport chain. These alterations included an up-regulation after surgery of ATP5G2, which encodes a subunit of the mitochondrial ATP synthase, and of $C O X 5 B$, the nuclear-encoded $\mathrm{Vb}$ subunit of the cytochrome c oxidase (COX) complex. The multi-subunit COX complex transfers electrons from cytochrome c (CYCS) 


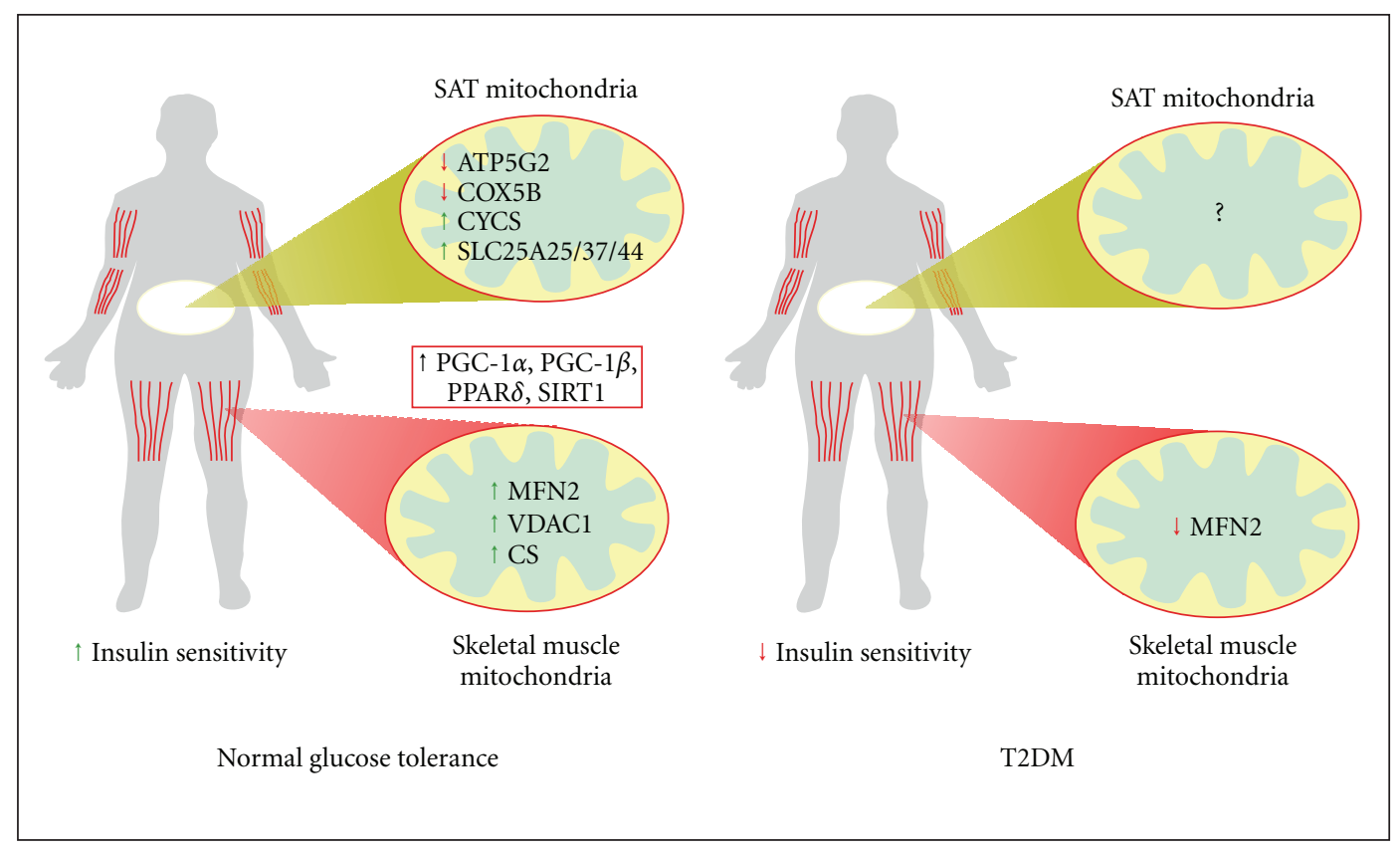

FIGURE 2: Summary of altered mitochondrial and mitochondrial biogenesis (boxed) gene expression in skeletal muscle of morbidly obese patients with normal glucose tolerance or type 2 diabetes mellitus (T2DM) [86] and in subcutaneous adipose tissue (SAT) one year after $\mathrm{BPD} / \mathrm{DS}$ surgery [49]. Arrows indicate the direction of the altered gene expression after bariatric surgery.

to molecular oxygen to form water. The expression of CYCS, on the other hand, which is also an intermediate in apoptosis, was down-regulated in adipose tissue after bariatric surgery. In addition, three genes of the solute carrier family (SLC25A25, SLC25A37, SLC25A44), involved in shuttling phosphate, iron, and adenine nucleotides across the inner mitochondrial membrane, were significantly down-regulated postsurgery. Another study investigated alterations in skeletal muscle mitochondrial function two years after BPD surgery, comparing morbidly obese patients to patients with normal glucose tolerance (NGT) and T2DM [86]. It was demonstrated that BPD surgery increased insulin sensitivity in both NGT and T2DM patients, an effect that did not correlate with induced mitochondrial gene expression in the diabetic patients (Figure 2). NGT patients showed an increased expression of genes regulating mitochondrial biogenesis in skeletal muscle, including PGC-1 $\alpha, P G C-1 \beta, P P A R \delta$, and SIRT1 (a gene that regulates PGC- $1 \alpha$ expression in liver and muscle) [86]. The expression of other mitochondrial genes such as MFN2 and the constitutive genes porin (VDAC1) and citrate synthase $(C S)$ were also significantly increased in the NGT patients. In the diabetic patients, no change was observed with the exception of MFN2 which was downregulated. Moreover, a differential oxidative profile in line with the mitochondrial gene expression could be observed in the two groups. Glucose oxidation during fasting was higher in the NGT group and lipid oxidation was higher in the diabetic group after BPD surgery. These results suggest a differential regulation of mitochondrial function in response to BPD in patients with NGT and T2DM, respectively.

\section{Future Perspectives}

Many aspects of the metabolic effects of bariatric surgery remain inadequately addressed or unanswered. There is convincing evidence that bariatric surgery exerts acute effects on metabolism that are independent of energy restriction and fat loss. The role of incretins on the metabolic functions of the liver, adipose tissue, and skeletal muscle should be further investigated. Molecular biology tools including global gene expression analysis and proteomics should be applied on tissue biopsies and isolated cell fractions collected before and shortly after bariatric surgery. Since certain biopsies are difficult to obtain from humans (e.g., postoperative visceral fat), the rat may be a useful model for studying the acute as well as long-term metabolic effects of bariatric surgery in all tissues [88-90]. In humans, short-term randomized controlled trials with increased power and direct comparison of different surgical procedures should be performed, and these would be strengthened by simultaneous measurement of tissue-specific metabolic parameters. More detailed analysis of parameters such as mitochondrial function may reveal novel mechanisms that may be targeted for more successful treatment of obesity and related diseases. The acute and long-term effects of specific bariatric surgery procedures on tissue and mitochondrial functions should be further investigated.

\section{Acknowledgments}

This work was supported by a Grant from NordForsk, Grant no. 070010, MitoHealth (to L. Burri and R. K. Berge), and by funding from the Research Council of Norway 
(RCN), Samarbeidsorganet Helse Vest RHF, Meltzerfondet, and Programstyret for ernæring at the University of Bergen.

\section{References}

[1] H. Buchwald, R. Estok, K. Fahrbach et al., "Weight and type 2 diabetes after bariatric surgery: systematic review and metaanalysis," American Journal of Medicine, vol. 122, no. 3, pp. 248-e5, 2009.

[2] C. Martins, M. Strømmen, O. A. Stavne, R. Nossum, R. Mårvik, and B. Kulseng, "Bariatric surgery versus lifestyle interventions for morbid obesity-changes in body weight, risk factors and comorbidities at 1 year," Obesity Surgery. In press.

[3] C. P. Cannon and A. Kumar, "Treatment of overweight and obesity: lifestyle, pharmacologic, and surgical options," Clinical Cornerstone, vol. 9, no. 4, pp. 55-71, 2009.

[4] W. J. Pories, K. G. MacDonald, E. J. Morgan et al., "Surgical treatment of obesity and its effect on diabetes: 10-y followup," American Journal of Clinical Nutrition, vol. 55, no. 2, pp. 582S-585S, 1992.

[5] P. R. Schauer, B. Burguera, S. Ikramuddin et al., "Effect of laparoscopic Roux-en Y gastric bypass on type 2 diabetes mellitus," Annals of Surgery, vol. 238, no. 4, pp. 467-485, 2003.

[6] M. H. Ahmed and C. D. Byrne, "Bariatric surgery and renal function: a precarious balance between benefit and harm," Nephrology Dialysis Transplantation, vol. 25, no. 10, pp. 31423147, 2010.

[7] P. Shankar, M. Boylan, and K. Sriram, "Micronutrient deficiencies after bariatric surgery," Nutrition, vol. 26, no. 11-12, pp. 1031-1037, 2010.

[8] P. E. O’Brien, T. McPhail, T. B. Chaston, and J. B. Dixon, "Systematic review of medium-term weight loss after bariatric operations," Obesity Surgery, vol. 16, no. 8, pp. 1032-1040, 2006.

[9] D. S. Hess, D. W. Hess, and R. S. Oakley, "The biliopancreatic diversion with the duodenal switch: results beyond 10 years," Obesity Surgery, vol. 15, no. 3, pp. 408-416, 2005.

[10] D. E. Cummings, "Endocrine mechanisms mediating remission of diabetes after gastric bypass surgery," International Journal of Obesity, vol. 33, no. 1, pp. S33-S40, 2009.

[11] F. Fery, N. P. D’Attellis, and E. O. Balasse, "Mechanisms of starvation diabetes: a study with double tracer and indirect calorimetry," American Journal of Physiology, vol. 259, no. 6, pp. E770-E777, 1990.

[12] R. R. Wing, E. H. Blair, P. Bononi, M. D. Marcus, R. Watanabe, and R. N. Bergman, "Caloric restriction per se is a significant factor in improvements in glycemic control and insulin sensitivity during weight loss in obese NIDDM patients," Diabetes Care, vol. 17, no. 1, pp. 30-36, 1994.

[13] T. A. Hughes, J. T. Gwynne, and B. R. Switzer, "Effects of caloric restriction and weight loss on glycemic control, insulin release and resistance, and atherosclerotic risk in obese patients with type II diabetes mellitus," American Journal of Medicine, vol. 77, no. 1, pp. 7-17, 1984.

[14] T. P. Markovic, A. B. Jenkins, L. V. Campbell, S. M. Furier, E. W. Kraegen, and D. J. Chisholm, "The determinants of glycemic responses to diet restriction and weight loss in obesity and NIDDM," Diabetes Care, vol. 21, no. 5, pp. 687694,1998

[15] K. Wickremesekera, G. Miller, T. DeSilva Naotunne, G. Knowles, and R. S. Stubbs, "Loss of insulin resistance after Roux-en-Y gastric bypass surgery: a time course study," Obesity Surgery, vol. 15, no. 4, pp. 474-481, 2005.
[16] J. B. Dixon, P. E. O’Brien, J. Playfair et al., "Adjustable gastric banding and conventional therapy for type 2 diabetes: a randomized controlled trial," Journal of the American Medical Association, vol. 299, no. 3, pp. 316-323, 2008.

[17] S. R. Kashyap, S. Daud, K. R. Kelly et al., "Acute effects of gastric bypass versus gastric restrictive surgery on $\beta$ cell function and insulinotropic hormones in severely obese patients with type 2 diabetes," International Journal of Obesity, vol. 34, no. 3, pp. 462-471, 2010.

[18] R. Peterli, B. Wölnerhanssen, T. Peters et al., "Improvement in glucose metabolism after bariatric surgery: comparison of laparoscopic roux-en-Y gastric bypass and laparoscopic sleeve gastrectomy: a prospective randomized trial," Annals of Surgery, vol. 250, no. 2, pp. 234-241, 2009.

[19] C. Guidone, M. Manco, E. Valera-Mora et al., "Mechanisms of recovery from type 2 diabetes after malabsorptive bariatric surgery," Diabetes, vol. 55, no. 7, pp. 2025-2031, 2006.

[20] S. Salinari, C. Guidone, A. Bertuzzi, M. Manco, S. Asnaghi, and G. Mlngrone, "First-phase insulin secretion restoration and differential response to glucose load depending on the route of administration in type 2 diabetic subjects after bariatric Surgery," Diabetes Care, vol. 32, no. 3, pp. 375-380, 2009.

[21] G. M. Campos, C. Rabl, S. Peeva et al., "Improvement in peripheral glucose uptake after gastric bypass surgery is observed only after substantial weight loss has occurred and correlates with the magnitude of weight lost," Journal of Gastrointestinal Surgery, vol. 14, no. 1, pp. 15-23, 2009.

[22] M. M. O. Lima, J. C. Pareja, S. M. Alegre et al., "Acute effect of Roux-en-Y gastric bypass on whole-body insulin sensitivity: a study with the euglycemic-hyperinsulinemic clamp," Journal of Clinical Endocrinology and Metabolism, vol. 95, no. 8, pp. 3871-3875, 2010.

[23] B. Laferrère, J. Teixeira, J. McGinty et al., "Effect of weight loss by gastric bypass surgery versus hypocaloric diet on glucose and incretin levels in patients with type 2 diabetes," Journal of Clinical Endocrinology and Metabolism, vol. 93, no. 7, pp. 2479-2485, 2008.

[24] G. J. Service, G. B. Thompson, F. J. Service, J. C. Andrews, M. L. Collazo-Clavell, and R. V. Lloyd, "Hyperinsulinemic hypoglycemia with nesidioblastosis after gastric-bypass surgery," The New England Journal of Medicine, vol. 353, no. 3, pp. 249254, 2005.

[25] T. Carpenter, M. E. Trautmann, A. D. Baron et al., "Hyperinsulinemic hypoglycemia with nesidioblastosis after gastricbypass surgery," The New England Journal of Medicine, vol. 353, no. 20, pp. 2192-2194, 2005.

[26] S. Troy, M. Soty, L. Ribeiro et al., "Intestinal gluconeogenesis is a key factor for early metabolic changes after gastric bypass but not after gastric lap-band in mice," Cell Metabolism, vol. 8, no. 3, pp. 201-211, 2008.

[27] B. Laferrère, S. Heshka, K. Wang et al., "Incretin levels and effect are markedly enhanced 1 month after Roux-en-Y gastric bypass surgery in obese patients with type 2 diabetes," Diabetes Care, vol. 30, no. 7, pp. 1709-1716, 2007.

[28] D. J. Drucker, "The role of gut hormones in glucose homeostasis," Journal of Clinical Investigation, vol. 117, no. 1, pp. 24-32, 2007.

[29] D. Sandoval, "CNS GLP-1 regulation of peripheral glucose homeostasis," Physiology and Behavior, vol. 94, no. 5, pp. 670674, 2008.

[30] C. Yamada, Y. Yamada, K. Tsukiyama et al., "Genetic inactivation of GIP signaling reverses aging-associated insulin 
resistance through body composition changes," Biochemical and Biophysical Research Communications, vol. 364, no. 1, pp. 175-180, 2007.

[31] F. Rubino, P. Zizzari, C. Tomasetto et al., "The role of the small bowel in the regulation of circulating ghrelin levels and food intake in the obese Zucker rat," Endocrinology, vol. 146, no. 4, pp. 1745-1751, 2005.

[32] F. Rubino, A. Forgione, D. E. Cummings et al., "The mechanism of diabetes control after gastrointestinal bypass surgery reveals a role of the proximal small intestine in the pathophysiology of type 2 diabetes," Annals of Surgery, vol. 244, no. 5, pp. 741-749, 2006.

[33] L. Rodriguez-Grunert, M. P. Galvao Neto, M. Alamo, A. C. Ramos, P. B. Baez, and M. Tarnoff, "First human experience with endoscopically delivered and retrieved duodenal-jejunal bypass sleeve," Surgery for Obesity and Related Diseases, vol. 4, no. 1, pp. 55-59, 2008.

[34] J. G. Kral, W. Paez, and B. M. Wolfe, "Vagal nerve function in obesity: therapeutic implications," World Journal of Surgery, vol. 33, no. 10, pp. 1995-2006, 2009.

[35] M. Camilleri, J. Toouli, M. F. Herrera et al., "Selection of electrical algorithms to treat obesity with intermittent vagal block using an implantable medical device," Surgery for Obesity and Related Diseases, vol. 5, no. 2, pp. 224-229, 2009.

[36] H. R. Berthoud and B. Jeanrenaud, "Acute hyperinsulinemia and its reversal by vagotomy after lesions of the ventromedial hypothalamus in anesthetized rats," Endocrinology, vol. 105, no. 1, pp. 146-151, 1979.

[37] A. Pocai, S. Obici, G. J. Schwartz, and L. Rossetti, "A brain-liver circuit regulates glucose homeostasis," Cell Metabolism, vol. 1, no. 1, pp. 53-61, 2005.

[38] B. T. Bikman, D. Zheng, W. J. Pories et al., "Mechanism for improved insulin sensitivity after gastric bypass surgery," Journal of Clinical Endocrinology and Metabolism, vol. 93, no. 12, pp. 4656-4663, 2008.

[39] M. Noakes, P. R. Foster, J. B. Keogh, A. P. James, J. C. Mamo, and P. M. Clifton, "Comparison of isocaloric very low carbohydrate/high saturated fat and high carbohydrate/low saturated fat diets on body composition and cardiovascular risk," Nutrition and Metabolism, vol. 3, article 7, 2006.

[40] J. M. R. Gill and A. R. Cooper, "Physical activity and prevention of type 2 diabetes mellitus," Sports Medicine, vol. 38 , no. 10 , pp. 807-824, 2008.

[41] S. P. Poulos, D. B. Hausman, and G. J. Hausman, "The development and endocrine functions of adipose tissue," Molecular and Cellular Endocrinology, vol. 323, no. 1, pp. 2034, 2010.

[42] S. Virtue and A. Vidal-Puig, "It's not how fat you are, it's what you do with it that counts.," PLoS Biology, vol. 6, no. 9, article e237, 2008.

[43] H. E. Bays, J. M. González-Campoy, G. A. Bray et al., "Pathogenic potential of adipose tissue and metabolic consequences of adipocyte hypertrophy and increased visceral adiposity," Expert Review of Cardiovascular Therapy, vol. 6, no. 3, pp. 343-368, 2008.

[44] H. E. Bays, B. Laferrère, J. Dixon et al., "Adiposopathy and bariatric surgery: is 'sick fat' a surgical disease?" International Journal of Clinical Practice, vol. 63, no. 9, pp. 1285-1300, 2009.

[45] S. J. Kim, C. Nian, and C. H. S. McIntosh, "Activation of lipoprotein lipase by glucose-dependent insulinotropic polypeptide in adipocytes: a role for a protein kinase B, LKB1, and AMP-activated protein kinase cascade," Journal of Biological Chemistry, vol. 282, no. 12, pp. 8557-8567, 2007.
[46] N. Irwin and P. R. Flatt, "Evidence for beneficial effects of compromised gastric inhibitory polypeptide action in obesityrelated diabetes and possible therapeutic implications," Diabetologia, vol. 52, no. 9, pp. 1724-1731, 2009.

[47] M. F. Gregor, L. Yang, E. Fabbrini et al., "Endoplasmic reticulum stress is reduced in tissues of obese subjects after weight loss," Diabetes, vol. 58, no. 3, pp. 693-700, 2009.

[48] R. Cancello, C. Henegar, N. Viguerie et al., "Reduction of macrophage infiltration and chemoattractant gene expression changes in white adipose tissue of morbidly obese subjects after surgery-induced weight loss," Diabetes, vol. 54, no. 8, pp. 2277-2286, 2005.

[49] S. N. Dankel, D. J. Fadnes, A. K. Stavrum et al., "Switch from stress response to homeobox transcription factors in adipose tissue after profound fat loss," PloS One, vol. 5, no. 6, Article ID e11033, 2010.

[50] C. Henegar, J. Tordjman, V. Achard et al., "Adipose tissue transcriptomic signature highlights the pathological relevance of extracellular matrix in human obesity," Genome Biology, vol. 9, no. 1, article R14, 2008.

[51] I. Dahlman, K. Linder, E. A. Nordström et al., "Changes in adipose tissue gene expression with energy-restricted diets in obese women," American Journal of Clinical Nutrition, vol. 81, no. 6, pp. 1275-1285, 2005.

[52] M. Kolehmainen, T. Salopuro, U. S. Schwab et al., "Weight reduction modulates expression of genes involved in extracellular matrix and cell death: the GENOBIN study," International Journal of Obesity, vol. 32, no. 2, pp. 292-303, 2008.

[53] R. Taylor, "Pathogenesis of type 2 diabetes: tracing the reverse route from cure to cause," Diabetologia, vol. 51, no. 10, pp. 1781-1789, 2008.

[54] J. G. Leichman, E. B. Wilson, T. Scarborough et al., "Dramatic reversal of derangements in muscle metabolism and left ventricular function after bariatric surgery," American Journal of Medicine, vol. 121, no. 11, pp. 966-973, 2008.

[55] A. V. Greco, G. Mingrone, A. Giancaterini et al., "Insulin resistance in morbid obesity: reversal with intramyocellular fat depletion," Diabetes, vol. 51, no. 1, pp. 144-151, 2002.

[56] G. F. Adami, R. C. Parodi, F. Papadia et al., "Magnetic resonance spectroscopy facilitates assessment of intramyocellular lipid changes: a preliminary short-term study following biliopancreatic diversion," Obesity Surgery, vol. 15, no. 9, pp. 1233-1237, 2005.

[57] L. Johansson, M. Roos, J. Kullberg et al., "Lipid mobilization following Roux-en-Y gastric bypass examined by magnetic resonance imaging and spectroscopy," Obesity Surgery, vol. 18, no. 10, pp. 1297-1304, 2008.

[58] D. M. Muoio and C. B. Newgard, "Obesity-related derangements in metabolic regulation," Annual Review of Biochemistry, vol. 75, pp. 367-401, 2006.

[59] S. Shalhub, A. Parsee, S. F. Gallagher et al., "The importance of routine liver biopsy in diagnosing nonalcoholic steatohepatitis in bariatric patients," Obesity Surgery, vol. 14, no. 1, pp. 54-59, 2004.

[60] L. A. Adams, J. F. Lymp, J. St. Sauver et al., “The natural history of nonalcoholic fatty liver disease: a population-based cohort study," Gastroenterology, vol. 129, no. 1, pp. 113-121, 2005.

[61] E. C. Verna and P. D. Berk, "Role of fatty acids in the pathogenesis of obesity and fatty liver: impact of bariatric surgery," Seminars in Liver Disease, vol. 28, no. 4, pp. 407-426, 2008. 
[62] A. R. Moschen, C. Molnar, A. M. Wolf et al., "Effects of weight loss induced by bariatric surgery on hepatic adipocytokine expression," Journal of Hepatology, vol. 51, no. 4, pp. 765-777, 2009.

[63] L. N. Bell, C. J. Temm, R. Saxena et al., "Bariatric surgeryinduced weight loss reduces hepatic lipid peroxidation levels and affects hepatic cytochrome P-450 protein content," Annals of Surgery, vol. 251, no. 6, pp. 1041-1048, 2010.

[64] N. C. Chavez-Tapia, F. I. Tellez-Avila, T. Barrientos-Gutierrez, N. Mendez-Sanchez, J. Lizardi-Cervera, and M. Uribe, "Bariatric surgery for non-alcoholic steatohepatitis in obese patients," Cochrane Database of Systematic Reviews, no. 1, Article ID CD007340, 2010.

[65] K. E. Wellen and G. S. Hotamisligil, "Inflammation, stress, and diabetes," Journal of Clinical Investigation, vol. 115, no. 5, pp. 1111-1119, 2005.

[66] R. Boushel, E. Gnaiger, P. Schjerling, M. Skovbro, R. Kraunsøe, and F. Dela, "Patients with type 2 diabetes have normal mitochondrial function in skeletal muscle," Diabetologia, vol. 50, no. 4, pp. 790-796, 2007.

[67] D. E. Kelley, J. He, E. V. Menshikova, and V. B. Ritov, "Dysfunction of mitochondria in human skeletal muscle in type 2 diabetes," Diabetes, vol. 51, no. 10, pp. 2944-2950, 2002.

[68] J. O. Holloszy, "Skeletal muscle "mitochondrial deficiency" does not mediate insulin resistance," American Journal of Clinical Nutrition, vol. 89, no. 1, pp. 463S-466S, 2009.

[69] M. A. Abdul-Ghani and R. A. DeFronzo, "Mitochondrial dysfunction, insulin resistance, and type 2 diabetes mellitus," Current Diabetes Reports, vol. 8, no. 3, pp. 173-178, 2008.

[70] J. Szendroedi and M. Roden, "Mitochondrial fitness and insulin sensitivity in humans," Diabetologia, vol. 51, no. 12, pp. 2155-2167, 2008.

[71] I. Pagel-Langenickel, J. Bao, L. Pang, and M. N. Sack, "The role of mitochondria in the pathophysiology of skeletal muscle insulin resistance," Endocrine Reviews, vol. 31, no. 1, pp. 25-51, 2010.

[72] K. F. Petersen, D. Befroy, S. Dufour et al., "Mitochondrial dysfunction in the elderly: possible role in insulin resistance," Science, vol. 300, no. 5622, pp. 1140-1142, 2003.

[73] M. Mogensen, K. Sahlin, M. Fernström et al., "Mitochondrial respiration is decreased in skeletal muscle of patients with type 2 diabetes," Diabetes, vol. 56, no. 6, pp. 1592-1599, 2007.

[74] V. B. Ritov, E. V. Menshikova, J. He, R. E. Ferrell, B. H. Goodpaster, and D. E. Kelley, "Deficiency of subsarcolemmal mitochondria in obesity and type 2 diabetes," Diabetes, vol. 54, no. 1, pp. 8-14, 2005.

[75] K. S. Nair, M. L. Bigelow, Y. W. Asmann et al., "Asian indians have enhanced skeletal muscle mitochondrial capacity to produce ATP in association with severe insulin resistance," Diabetes, vol. 57, no. 5, pp. 1166-1175, 2008.

[76] H. M. de Feyter, N. M. A. van den Broek, S. F. E. Praet, K. Nicolay, L. J. C. van Loon, and J. J. Prompers, "Early or advanced stage type 2 diabetes is not accompanied by in vivo skeletal muscle mitochondrial dysfunction," European Journal of Endocrinology, vol. 158, no. 5, pp. 643-653, 2008.

[77] O. Shaham, RU. Wei, T. J. Wang et al., "Metabolic profiling of the human response to a glucose challenge reveals distinct axes of insulin sensitivity," Molecular Systems Biology, vol. 4, article 214, 2008.

[78] C. S. Stump, K. R. Short, M. L. Bigelow, J. M. Schimke, and K. S. Nair, "Effect of insulin on human skeletal muscle mitochondrial ATP production, protein synthesis, and mRNA transcripts," Proceedings of the National Academy of Sciences of the United States of America, vol. 100, no. 13, pp. 7996-8001, 2003.

[79] V. K. Mootha, C. M. Lindgren, K. F. Eriksson et al., "PGC$1 \alpha$-responsive genes involved in oxidative phosphorylation are coordinately downregulated in human diabetes," Nature Genetics, vol. 34, no. 3, pp. 267-273, 2003.

[80] M. E. Patti, A. J. Butte, S. Crunkhorn et al., "Coordinated reduction of genes of oxidative metabolism in humans with insulin resistance and diabetes: potential role of PGC1 and NRF1," Proceedings of the National Academy of Sciences of the United States of America, vol. 100, no. 14, pp. 8466-8471, 2003.

[81] G. P. Holloway, C. G. R. Perry, A. B. Thrush et al., "PGC-1 $\alpha$ 's relationship with skeletal muscle palmitate oxidation is not present with obesity despite maintained PGC- $1 \alpha$ and PGC- $1 \beta$ protein," American Journal of Physiology, vol. 294, no. 6, pp. E1060-E1069, 2008.

[82] D. Bach, D. Naon, S. Pich et al., "Expression of Mfn2, the Charcot-Marie-Tooth neuropathy type 2A gene, in human skeletal muscle: effects of type 2 diabetes, obesity, weight loss, and the regulatory role of tumor necrosis factor $\alpha$ and interleukin-6," Diabetes, vol. 54, no. 9, pp. 2685-2693, 2005.

[83] D. Bach, S. Pich, F. X. Soriano et al., "Mitofusin-2 determines mitochondrial network architecture and mitochondrial metabolism: a novel regulatory mechanism altered in obesity," Journal of Biological Chemistry, vol. 278, no. 19, pp. 1719017197, 2003.

[84] K. H. Chen, X. Guo, D. Ma et al., "Dysregulation of HSG triggers vascular proliferative disorders," Nature Cell Biology, vol. 6, no. 9, pp. 872-883, 2004.

[85] O. M. de Brito and L. Scorrano, "Mitofusin 2 tethers endoplasmic reticulum to mitochondria," Nature, vol. 456, no. 7222, pp. 605-610, 2008.

[86] M. I. Hernández-Alvarez, C. Chiellini, M. Manco et al., "Genes involved in mitochondrial biogenesis/function are induced in response to bilio-pancreatic diversion in morbidly obese individuals with normal glucose tolerance but not in type 2 diabetic patients," Diabetologia, vol. 52, no. 8, pp. 1618-1627, 2009.

[87] I. Dahlman, M. Forsgren, A. Sjögren et al., "Downregulation of electron transport chain genes in visceral adipose tissue in type 2 diabetes independent of obesity and possibly involving tumor necrosis factor- $\alpha$," Diabetes, vol. 55 , no. 6 , pp. $1792-$ 1799, 2006.

[88] Y. Xu, K. Ohinata, M. M. Meguid et al., "Gastric bypass model in the obese rat to study metabolic mechanisms of weight loss," Journal of Surgical Research, vol. 107, no. 1, pp. 56-63, 2002.

[89] H. Kirchner, A. Guijarro, and M. M. Meguid, "Is a model useful in exploring the catabolic mechanisms of weight loss after gastric bypass in humans?" Current Opinion in Clinical Nutrition and Metabolic Care, vol. 10, no. 4, pp. 463-474, 2007.

[90] E. Nadreau, E. D. Baraboi, P. Samson et al., "Effects of the biliopancreatic diversion on energy balance in the rat," International Journal of Obesity, vol. 30, no. 3, pp. 419-429, 2006. 


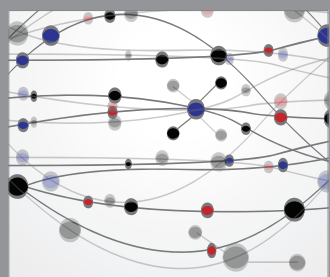

The Scientific World Journal
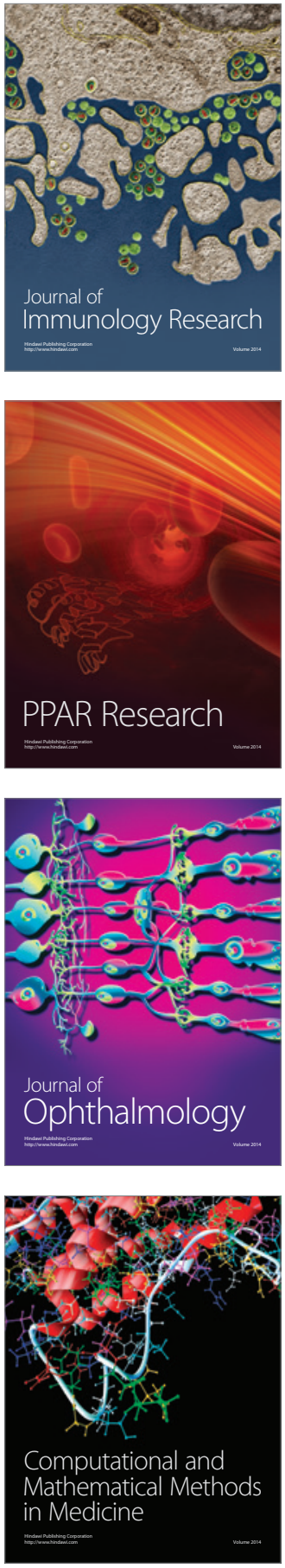

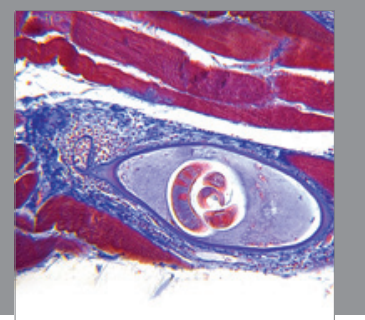

Gastroenterology

Research and Practice
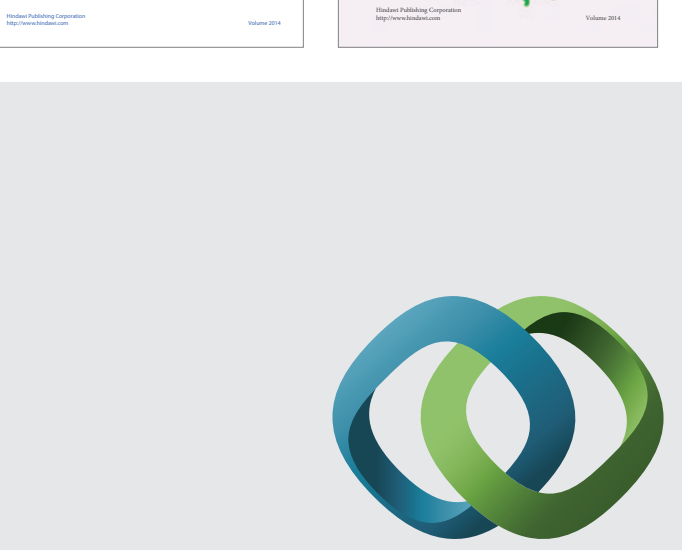

\section{Hindawi}

Submit your manuscripts at

http://www.hindawi.com
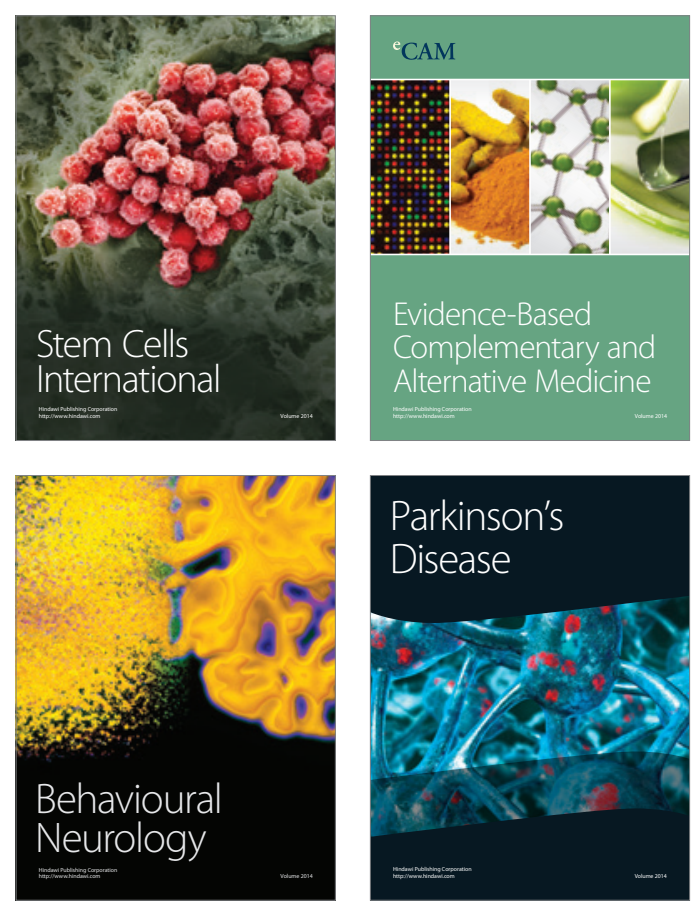

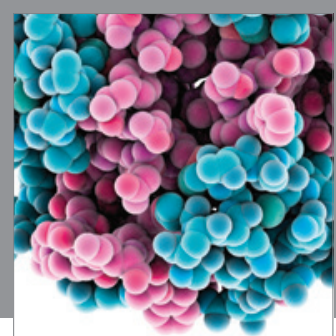

Journal of
Diabetes Research

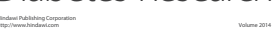

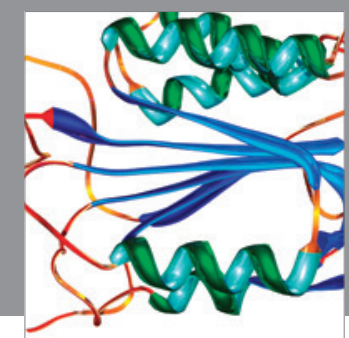

Disease Markers
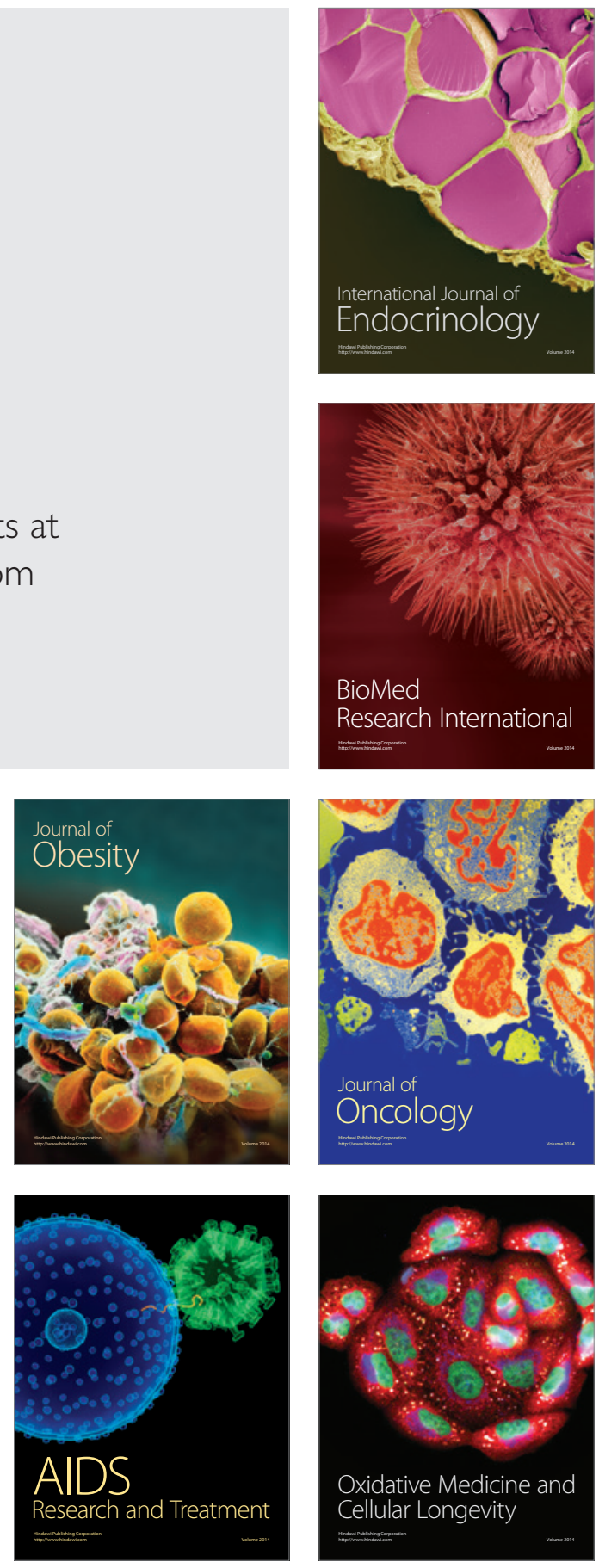